\title{
HALLEY'S COMET COMETH
}

STAN SHADICK, Department of Physics, University of Saskatchewan, Saskatoon, Saskatchewan. STN OWO

For most people their once in a lifetime opportunity to view Halley's Comet will come this winter and spring. Very few persons can claim to have sighted this famous comet when it last appeared in 1910.

Halley's Comet is believed to be a solid nucleus of dust and ice only about $7 \mathrm{~km}$ in size. Unlike the circular orbits of the planets, comets move in very elliptical orbits. Halley's Comet spends most of its time out beyond the orbit of Jupiter. Once every 76 years it hurtles around the sun inside the orbit of the earth. At that time the solar radiation vaporises part of the nucleus, producing tails of gas and dust.

Astronomers have traced historical references of Halley's Comet back to 240 B.C. A few of its appearances have coincided with military defeats such as the destruction of Jerusalem in 66, the defeat of Attila the Hun in 451 and the Battle of Hastings in 1066. Such events have caused some people to view comets as ill omens.

During its last appearance in 1910, astronomers predicted that the earth would pass through the tail of the comet. This news caused some sensationalist newspaper reporters to predict that the atmosphere would become polluted with cyanogen. Swindlers had a field day in some areas selling comet pills and bottled oxygen. Although the comet put on a brilliant display, there was no evidence of pollution.

For its upcoming appearance, Comet Halley will pass closest to the sun on February 9, 1986. Unfortunately the long tail will not be visible then as the comet will be on the opposite side of the sun as seen from earth. In fact the geometry of the or- bit will likely produce one of the poorest shows on record -- especially for Canadian observers.

Nevertheless there will be times when Halley's Comet will be visible to the naked eye. It will be necessary to leave the background light pollution of our cities and towns and journey into some dark rural location. For the best view, be sure to take a pair of binoculars and choose a night when there is no moon.

The actual brightness of a comet can be very difficult to predict, as was evidenced by the infamous Comet Kohoutek. However astronomers now predict that Comet Halley should become visible as a faint, fuzzy, starlike image during December and January. The best time to look for the comet will be between 7:00 p.m. and 8:00 p.m. from 30 December until about 20 January. After that date the comet will be quickly approaching the sun and will become lost in the twilight.

The area in the sky in which to look for Halley's Comet is shown in Fig. 1. The chart shows the southwestern night sky at 7:00 p.m. on 30 December. Although it is drawn for an observer near Saskatoon, it can be used by any one located between latitudes $40^{\circ}$ and $60^{\circ}$ (i.e. most of Canada). Letters locate the positions of some reference stars as well as the planet Jupiter.

Throughout late December and January, Halley's Comet will be found within the faint constellation Aquarius. To locate the comet on 30 December use the following procedure:

1) Locate the relatively bright star Markab 


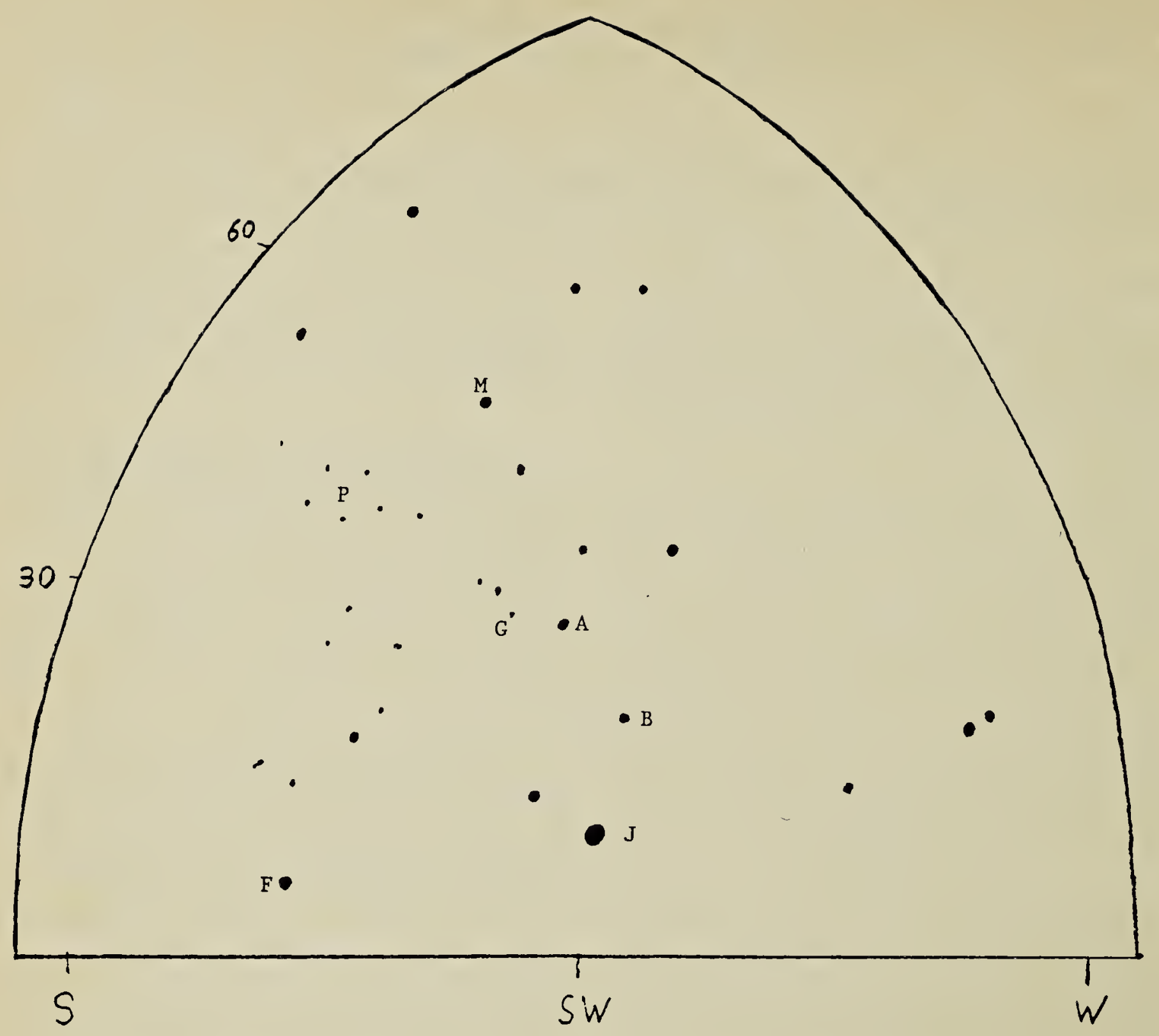

Figure 1. Saskatchewan night sky at 7 p.m. on 30 December 1985. Reference stars shown are: A -Alpha aquarius; B - Beta Aquarius; F - Formalhaut; $G$ - Gamma Aquarius; J - Planet Jupiter; $M$ - Markab; $P$ - Pentagon of stars in Pisces. Comet Halley appears near: P 12-15 December; $G 30$ December; A 4 January; $B 22$ January.

(labelled $M$ ) which is at the bottom corner of the Great Square in the constellation Pegasus

2) Locate the extremely bright planet Jupiter (labelled J) which is very low in the southwest

3) Approximately halfway between Markab and Jupiter you should find the star Alpha Aquarius (labelled A). It should appear almost as bright as Markab

4) A short distance to the left of Alpha Aquarius, locate a fainter star Gamma Aquarius (labelled G)
5) By scanning the sky with binoculars near Gamma Aquarius you should see Halley's Comet as a fuzzy object probably fainter than the star Gamma Aquarius. Be careful not to confuse it with the two other stars shown in the figure above Gamma Aquarius.

From night to night in early January, the comet should become slightly brighter but also move lower in the sky. By 20 January it should approach the vicinity of the star Beta Aquarius (labelled B).

It is possible that Halley's Comet may be visible earlier in December although it 
won't appear as bright. From 12-15 December try scanning with binoculars inside the pentagon of five stars (labelled P) to the lower left of the Great Square of Pegasus.

As the comet approaches the sun in February it will be too close to the sun to be seen. In March it should display an impressive tail for observers south of the southern United States. Here in Canada the tail will not appear as bright because it will be very close to the horizon.

To look for the comet's tail in March you will need to be south of the city and watch the southeastern horizon between 90 and $120 \mathrm{~min}$. before sunrise. To avoid problems with moonlight the best dates would be between 10 and 22 March. The exact time of sunrise varies depending upon the observer's location. But as a general rule the best time will be from 5:00 to 6:00 a.m. You should see the comet rise tail first, slanting up and to the right from a point on the horizon slightly to the north of southeast. Binoculars may help for a detailed view.

The comet will reappear late in the evening of 18 April low in the southern sky. However moonlight may make it difficult to find. Your last chance to avoid problems with moonlight and spot the comet will be about 11:00 p.m. on the last three days in April. Try looking about $15^{\circ}$ above the horizon in a SSW direction.

Readers interested in more information about Halley's Comet should consult articles in recent issues of the following magazines: Nature Canada (Oct/Dec 1982), Equinox (Mar 1985), Sky and Telescope, and Astronomy.

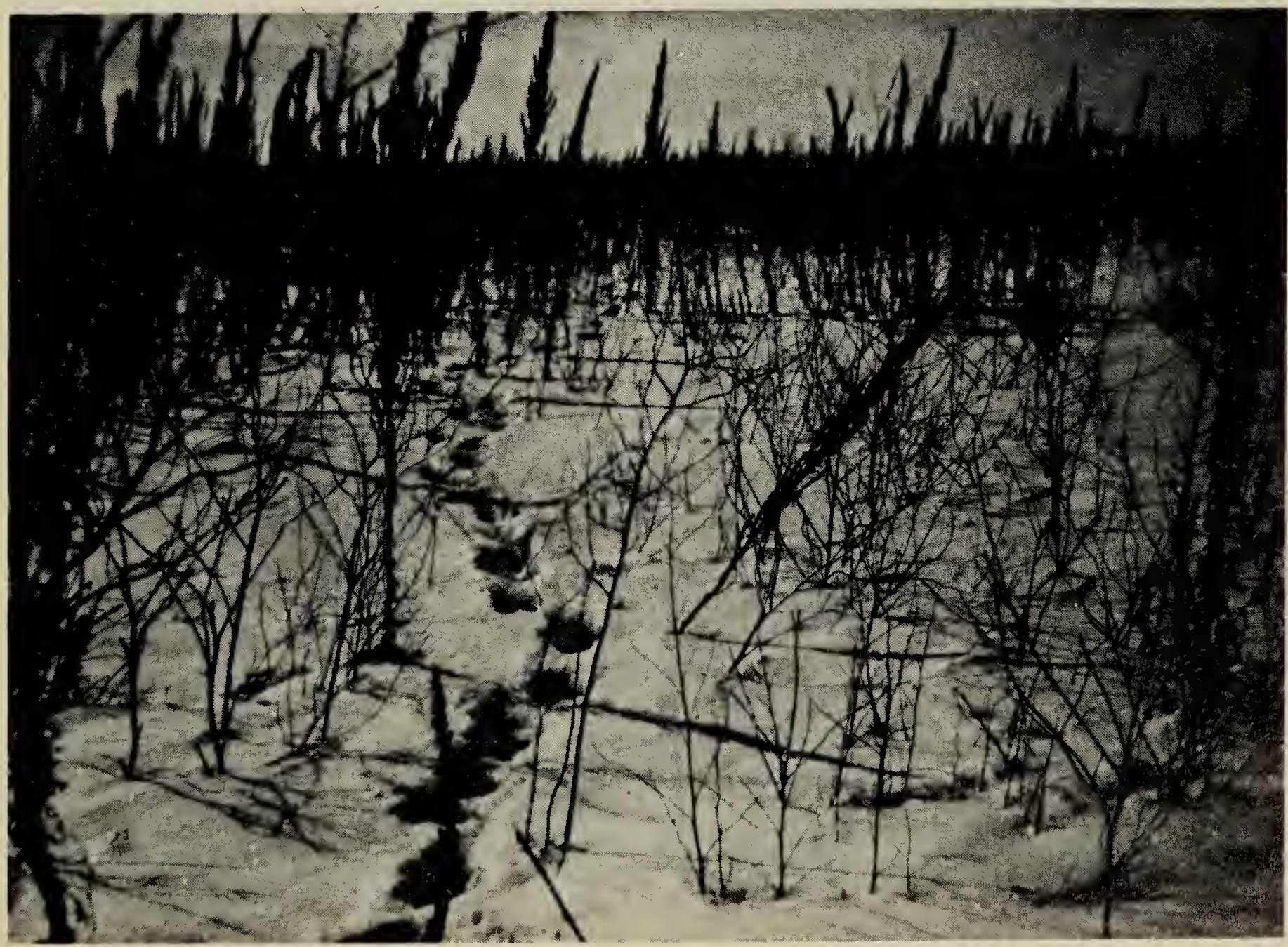

